\title{
Structural and Electronic Properties of Functionalized Graphene
}

\author{
K. Milowska, M. Birowska And J.A. Majewski \\ Institute of Theoretical Physics, Faculty of Physics, University of Warsaw \\ Hoża 69, PL-00-681 Warszawa, Poland
}

\begin{abstract}
In the present paper, we study the effects of functionalization of graphene with simple organic molecules $\mathrm{OH}$, and $\mathrm{NH}_{2}$, focusing on the stability and band gaps of the structures. We have performed DFT calculations for graphene supercells with various numbers of the attached molecules. We have determined adsorption energies of the functionalized graphene mono- and bilayers, the changes in the geometry, and the band structure. We observe the characteristic effects such as rehybridization of the bonds induced by fragments attached to graphene and opening of the graphene band gap by functionalization. We have also studied the dependence of the adsorption energies of the functionalized graphene on the density of the adsorbed molecules. Our calculations reveal that the $-\mathrm{OH}$ and $-\mathrm{NH}_{2}$ groups exhibit the strong cohesion to graphene layers. Further, we determine the critical density of the $\mathrm{OH}$ fragments which lead to the opening of the band gap. We also show how to engineer the magnitude of the band gap by functionalizing graphene with $\mathrm{NH}_{2}$ groups of various concentrations.
\end{abstract}

PACS: 31.15.E-, 61.46.-w, 73.20.At, 73.22.-f

\section{Introduction}

Remarkable electronic, mechanical and thermal properties of graphene have made it a promising candidate for a new generation of electronic devices $[1,2]$. However, the single and bilayer graphene have zero energy band gaps. Therefore, it hinders direct application of these systems in field effect transistors (FETs). On the other hand, the functionalization of the graphene could change its electronic structure. Since quantitatively this effect is rather unknown, we have undertaken theoretical studies of this issue. Some aspects of the cohesion in functionalized graphene monolayers have been studied previously [3-7]. We have performed extensive studies of the stability and electronic properties of the functionalized graphene with various molecular groups. In this letter, we focus on the graphene mono- and bilayers functionalized with the $-\mathrm{OH}$ and $-\mathrm{NH}_{2}$ fragments and report the results of $a b$ initio studies of their stability and band gap.

\section{Method}

Our studies of the graphene mono- and bilayers functionalized with $-\mathrm{OH}$ and $-\mathrm{NH}_{2}$ groups are based on the $a b$ initio calculations in the framework of the KohnSham realization of the density functional theory (DFT) $[8,9]$. We have used the generalized gradient approximation (GGA) for the exchange correlation density functional [10] and supercell geometry with periodic boundary conditions within the numerical package SIESTA
$[11,12]$. A kinetic energy cut-off of 500 Ry and split double zeta basis set with spin polarization have been used in all calculations. The lateral separation (i.e., lateral lattice constants in $z$ direction) has been set to $80 \AA$, just to eliminate completely the spurious interaction between neighboring cells. In $x y$ plane the system has full periodicity of the hexagonal lattice. We have performed calculations for six different supercells $1 \times 1,2 \times 2,3 \times 3$, $4 \times 4,5 \times 5,6 \times 6$, containing $2,8,18,32,50$, and 72 carbon atoms, respectively, and one functionalizing group attached to the graphene sheet. The calculations for the functionalized graphene bilayer were performed for the most stable AB (graphitic) stacking. The positions of all atoms in the supercell and the lattice parameters have been optimized. Hereafter, we present the results of the calculations for graphene mono- and bilayer functionalized with $-\mathrm{OH}$ and $-\mathrm{NH}_{2}$ groups.

\section{Results and discussion}

First of all, we analyze stability and change of structure mono- and bilayer graphene functionalized with $\mathrm{OH}$ and $\mathrm{NH}_{2}$. Both studied groups bind covalently to the graphene layer, implying $s p^{2}$ to $s p^{3}$ rehybridization and local deformation of the graphene plane. $\mathrm{C}-\mathrm{C}$ bond length in the closest proximity of the functionalizing group increases to $1.51 \AA$, whereas $\mathrm{C}-\mathrm{C}$ bond length is very close to the bond length in pure graphene (1.43 $\AA$ ), away from group. The carbon atom that is directly bonded to the group sticks out from the graphene sheet. We get very good agreement with existing experimental data $[6,7]$, and other theoretical calculations [3-5]. 
Interestingly, for the highest $(1 \times 1)$ concentrations of hydroxyl group, we observe separation of $\mathrm{O}$ and $\mathrm{H}$, which cover now both sides of the graphene layer.

The stability of all the structures has been analyzed in terms of adsorption energy calculated with respect to the following formula (1):

$$
\begin{aligned}
& E_{\text {ads }}=E_{\text {tot }}(\text { graphene }+ \text { group })-E_{\text {tot }}(\text { graphene }) \\
& -E_{\text {tot }}(\text { group }) .
\end{aligned}
$$

In Fig. 1, the adsorption energies of the graphene monolayer functionalized with $-\mathrm{OH}$ and $-\mathrm{NH}_{2}$ are depicted for various concentrations of functionalizing fragments, which is measured as the inverse of the number of carbon atoms per functionalizing group. It is clearly seen that both groups are strongly bound to the graphene surface. Adsorption energy is negative for all the cases, which means that all the structures are stable and their formation is energetically preferred. Generally, the stability of the functionalized graphene layers decreases slightly with the growing concentration of functionalizing molecules. In the case of the functionalized bilayer graphene, the stability of the functionalized structures is minimally larger than in the functionalized monolayer graphene (typically by the order of $10^{-2} \mathrm{eV}$ ).

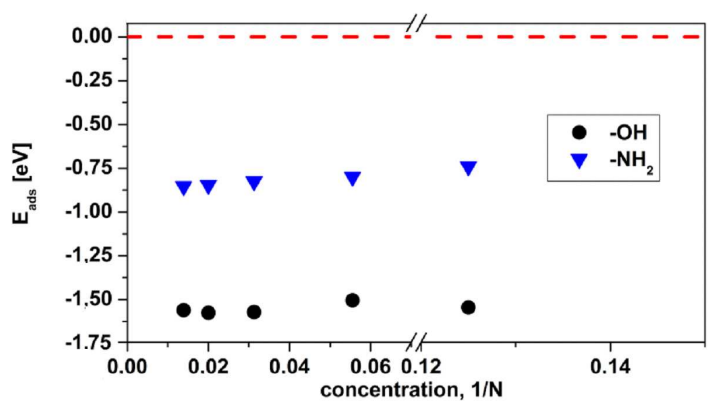

Fig. 1. Adsorption energies of functionalized graphene monolayer with hydroxyl $(\mathrm{OH})$ and amine $\left(\mathrm{NH}_{2}\right)$ groups as a function of the concentration of functionalizing units. The concentration is measured as the inverse of the number of carbon atoms $N$ per functionalizing group.

Next, we present results for the electronic structure of the functionalized graphene layers. In Fig. 2, some exemplary band structures of graphene monolayer functionalized with $\mathrm{OH}$ and $\mathrm{NH}_{2}$ are shown. The fundamental band gap has been calculated as the difference between the Kohn-Sham energy of highest occupied molecular orbital (HOMO) and the Kohn-Sham energy of the lowest unoccupied molecular orbital (LUMO). It turns out that the graphene monolayer functionalized with the $\mathrm{OH}$ group at low concentrations (see Fig. 2b) is semimetallic. However, the gap opens for high concentration of the functionalizing group, as depicted in Fig. 2a. The functionalization of the graphene monolayer with $\mathrm{NH}_{2}$ group leads always to the gap opening at all concentrations of the functionalizing group, as it is exemplified in Fig. 2c.

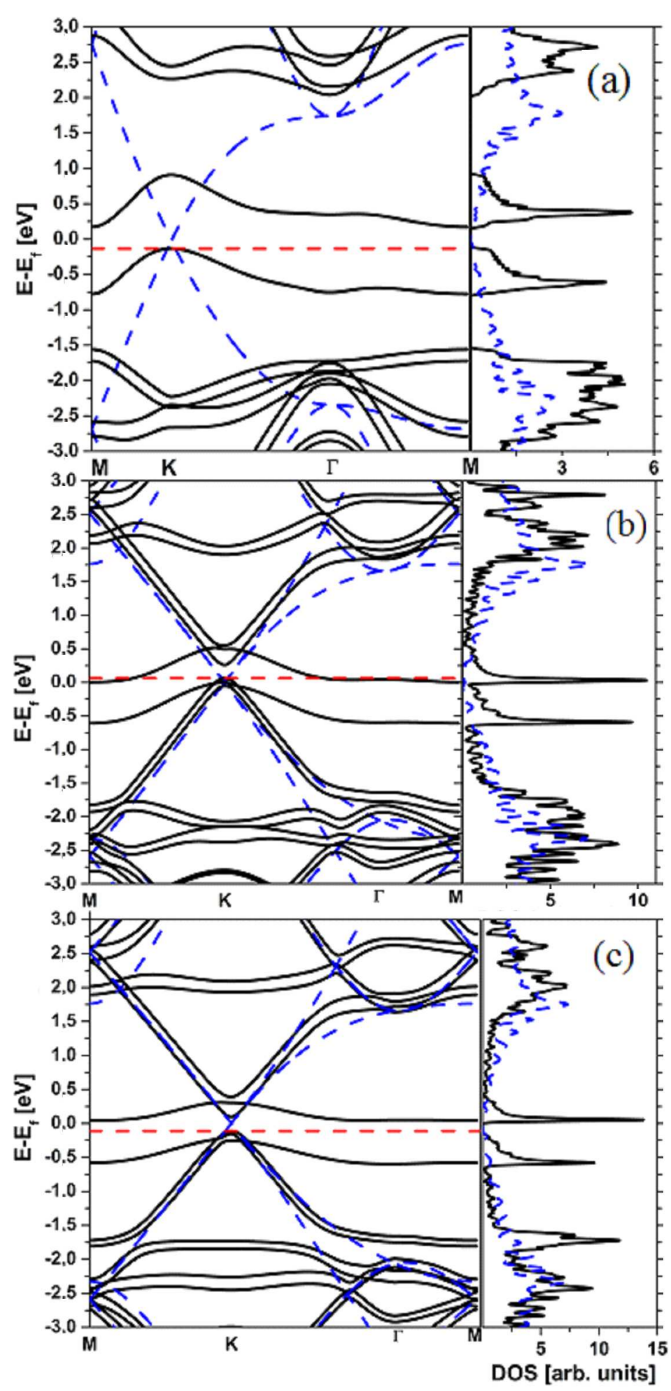

Fig. 2. Calculated band structure and density of states (DOS) of graphene monolayer functionalized with: (a) $\mathrm{OH}$ per 8 carbon atoms, (b) $\mathrm{OH}$ per 18 carbon atoms, (c) $\mathrm{NH}_{2}$ per 18 carbon atoms, plotted with black lines. For comparison, the band structure and DOS for pure graphene are depicted (blue lines). The dashed red line indicates the position of the HOMO in the functionalized structure. The graphene functionalized with $\mathrm{OH}$ group at low concentration (b) exhibits zero energy gap. The fundamental energy gap in the cases (a) and (c) is indirect, and is equal to $0.306 \mathrm{eV}$ and $0.154 \mathrm{eV}$ for the case (a) and (c), respectively.

The fundamental band gaps for the structures shown in Fig. 2 are indirect, and they are equal to $0.306 \mathrm{eV}, 0.0 \mathrm{eV}$, and $0.154 \mathrm{eV}$, for the case (a), (b), and (c), respectively, whereas the direct gap in the $K$-point is always positive, being equal to $1.04 \mathrm{eV}, 0.20 \mathrm{eV}$, and $0.21 \mathrm{eV}$ for cases (a), (b), and (c), respectively. In Fig. 2, the "impurity" bands originating from the states of the functionalizing groups are clearly seen. Actually, the band gap arises between bands originating from the bonding and antibonding states of the functionalizing molecules. For 
very high concentration of $\mathrm{OH}$ molecules (see Fig. 2a), interaction between $\mathrm{OH}$ groups causes radical changes in the band structure of the functionalized graphene in comparison to the pure one.

For design of functional devices, it is interesting to which extent the energy band gap can be engineered. It is demonstrated in Fig. 3, which presents the theoretical predictions of the band in the graphene monolayer functionalized with amine at various concentrations. Quite naturally, the band gap increases with the concentration of the attached molecules. All this shows clearly that the band gap of graphene layers can be tuned within fairly large range.

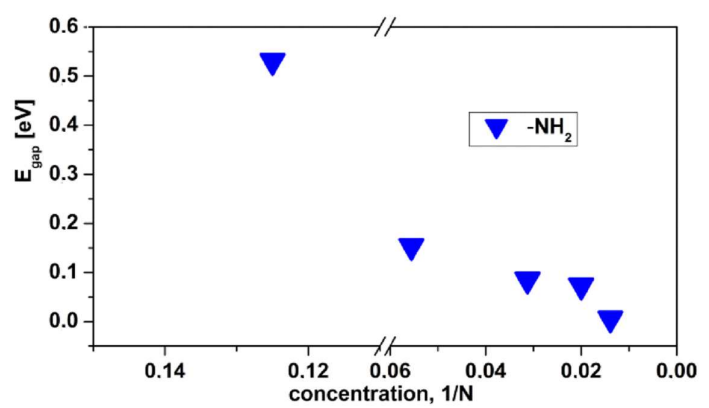

Fig. 3. Band gap energy for graphene monolayer functionalized with $\mathrm{NH}_{2}$ as a function of concentration of functionalizing molecules, measured as the number of carbon atoms $N$ per one molecule.

Qualitatively, analogous situation is observed in the case of functionalized graphene bilayer. However, the calculated band gaps are considerably smaller (typically by a factor of 10) than in the case of the functionalized graphene monolayer. As solution for this problem, we propose functionalization of the bilayer graphene by performing some kind of intercalation with the functionalizing hydroxyl and amine groups. Preliminary results are very promising and will be published elsewhere.

\section{Conclusions}

We have performed $a b$ initio studies of the stability and electronic structure of graphene mono- and bilayer functionalized with $-\mathrm{OH}$ and $-\mathrm{NH}_{2}$ groups.

Physical mechanism of the functionalization can be understood in terms of rehybridization of the bonds induced by adsorbed group.

The functionalization of graphene with hydroxyl and amine groups leads to the opening of the band gap, which in turn might be engineered by changing the concentration of the functionalizing group.

\section{Acknowledgments}

The studies were carried out within the SiCMAT Project financed under the European Funds for Regional Development (contract No. UDA-POIG.01.03.01$-14-155 / 09)$

\section{References}

[1] S. Park, R.S. Ruoff, Nature Nanotechnol. 4, 217 (2009).

[2] D. Pacile, J.C. Meyer, A. Fraile Rodriguez, M. Papagno, C. Gomez-Navarro, R.S. Sundaram, M. Burghard, K. Kern, C. Carbone, U. Kaiser, Carbon 49, 966 (2011).

[3] J.-A. Yan, M.Y. Chou, Phys. Rev. B 82, 125403 (2010).

[4] D.W. Boukhvalov, arXiv:1008.4263.

[5] D.W. Boukhvalov, M.I. Katsnelson, arXiv:1012.3821.

[6] H.B. Su, R.J. Nielsen, A.C.T. van Duin, W.A. Goddard, Phys. Rev. B 75, 134107 (2007).

[7] K.-J. Huang, D.-J. Niu, X. Liu, Z.-W. Wu, Y. Fan, Y.-F. Chang, Y.-Y. Wu, Electrochim. Acta 56, 2947 (2011).

[8] P. Hohenberg, W. Kohn, Phys. Rev. 136, 864 (1964).

[9] W. Kohn, L.J. Sham, Phys. Rev. 140, A1133 (1965).

[10] J.P. Perdew, K. Burke, M. Ernzerhof, Phys. Rev. Lett. 77, 3865 (1996).

[11] P. Ordejón, E. Artacho, J.M. Soler, Phys. Rev. B 53 , R10441 (1996).

[12] J.M. Soler, E. Artacho, J.D. Gale, A. García, J. Junquera, P. Ordejón, D. Sánchez-Portal, J. Phys., Condens. Matter 14, 2745 (2002). 